\title{
The inverse along a lower triangular matrix*
}

\author{
Xavier Mary ${ }^{a}$, Pedro Patrício ${ }^{b}$
}

\begin{abstract}
${ }^{a}$ Université Paris-Ouest Nanterre - La Défense, Laboratoire Modal'X, 200 avenuue de la république, 92000 Nanterre, France. email: xavier.mary@u-paris10.fr

${ }^{b}$ Departamento de Matemática e Aplicações, Universidade do Minho, 4710-057 Braga, Portugal.

email: pedro@math.uminho.pt
\end{abstract}

\begin{abstract}
In this paper, we investigate the recently defined notion of inverse along an element in the context of matrices over a ring. Precisely, we study the inverse of a matrix along a lower triangular matrix, under some conditions.
\end{abstract}

Keywords: Generalized inverse, inverse along an element, Dedekind-finite ring, Green's relations, rings

AMS classification: 15A09, 16E50

\section{Introduction}

In this paper, $R$ is a ring with identity. We say $a$ is (von Neumann) regular in $R$ if $a \in a R a$. A particular solution to $a x a=a$ is denoted by $a^{-}$, and the set of all such solutions is denoted by $a\{1\}$. Given $a^{-}, a^{=} \in a\{1\}$ then $x=a^{=} a a^{-}$satisfies $a x a=a, x a x=a$ simultaneously. Such a solution is called a reflexive inverse, and is denoted by $a^{+}$. The set of all reflexive inverses of $a$ is denoted by $a\{1,2\}$. Finally, $a$ is group invertible if there is $a^{\#} \in a\{1,2\}$ that commutes with $a$, and $a$ is Drazin invertible if $a^{k}$ is group invertible, for some non-negative integer $k$. This is equivalent to the existence of $a^{D} \in R$ such that $a^{k+1} a^{D}=a^{k}, a^{D} a a^{D}=a^{D}, a a^{D}=a^{D} a$.

We say $R$ is a Dedekind-finite ring if $a b=1$ is sufficient for $b a=1$. This is equivalent to saying invertible lower triangular matrices are exactly the matrices whose diagonal elements are ring units, and in this case the matrix inverse is again lower triangular.

We will make use of the Green's relation $\mathcal{H}$ in $R$, see [5], defined by

$$
a \mathcal{H} b \text { if } a R=b R \text { and } R a=R b .
$$

$b \leq_{\mathcal{H}} d$ denotes $b \in d R \cap R d$.

In this paper, we will study invertibility along a fixed element, as defined recently in [12] in the context of semigroups.

\footnotetext{
${ }^{*}$ This research was financed by FEDER Funds through "Programa Operacional Factores de Competitividade - COMPETE" and by Portuguese Funds through FCT - "Fundação para a Ciência e a Tecnologia", within the project PEst-C/MAT/UI0013/2011.
} 
Definition 1.1. Given $a, d$ in $R$, we say $a$ is invertible along $d$ if there exists $b$ such that $b a d=d=d a b$ and $b \leq_{\mathcal{H}} d$. If such an element exists the it is unique and is denoted by $a^{\| d}$.

The inverse along an element reduces to von Neumann, group and Drazin inverses (see [12]) by $a^{\| 1}=a^{-1}, a^{\| a}=a^{\#}, a^{\| a^{k}}=a^{D}$.

In this paper, the existence of $a^{\| d}$ by means of a unit in the ring $R$ as studied in [13] will allow us to study invertibility of some matrices along lower triangular matrices. We will give an alternative proof for the sake of completness. In order to do so, we state a well known preliminary result.

Lemma 1.2 (Jacobson). $1-x y$ is a unit if and only if $1-y x$ is a unit, in which case $(1-x y)^{-1}=1+x(1-y x)^{-1} y$.

We refer the reader to [3] and [4] for a similar result with Drazin inverses.

Theorem 1.3. Let $a, d \in R$ such that $d$ is a regular element of a ring $R$, and let $d^{-} \in d\{1\}$. Then the following are equivalent:

1. $a^{\| d}$ exists.

2. $u=d a+1-d d^{-}$is a unit.

3. $v=a d+1-d^{-} d$ is a unit.

In this case,

$$
\begin{aligned}
a^{\| d} & =u^{-1} d \\
& =d v^{-1} .
\end{aligned}
$$

Proof. (2) and (3) are equivalent by writing $u=1-d\left(d^{-}-a\right), v=1-\left(d^{-}-a\right) d$ and applying Lemma 1.2.

Suppose now $a^{\| d}$ exists, that is, there is $b \in R$ such that $b a d=d=d a b$ with $b=d x=y d$, for some $x, y \in R$. Since $\left(d a d d^{-}+1-d d^{-}\right)\left(d x d^{-}+1-d d^{-}\right)=1=\left(y d d^{-}+1-d d^{-}\right)\left(d a d d^{-}+\right.$ $\left.1-d d^{-}\right)$then $d a d d^{-}+1-d d^{-}$is a ring unit. Note that we can write $u=d d^{-} d a+1-d d^{-}=$ $1+d d^{-}(1-d a)$ and therefore $u$ is a unit if and only if $d a d d^{-}+1-d d^{-}=1-(1-d a) d d^{-}$is a unit, using Lemma 1.2.

Conversely, suppose $u$, and therefore, $v$ are units. Since $u d=d a d=d v$ then $u^{-1} d=d v^{-1}$ and $d=\left(u^{-1} d\right) a d=d a\left(d v^{-1}\right)$. Taking $b=u^{-1} d=d v^{-1}$ then obviously $b \in R d \cap d R$. Therefore $a^{\| d}=b=u^{-1} d=d v^{-1}$.

The previous theorem shows, in particular, that given $d$ regular then $1^{\| d}$ exists if and only if $d^{\#}$ exists, using [18] and Lemma 1.2. 


\section{The inverse of a lower triangular matrix along another lower triangular matrix}

Let $D$ be a regular lower triangular matrix and suppose $B=A^{\| D}$ exists, with $A$ lower triangular. Write $D=\left[\begin{array}{cc}d_{1} & 0 \\ d_{2} & d_{3}\end{array}\right]$ and $A=\left[\begin{array}{cc}a & 0 \\ b & d\end{array}\right]$. According to [14], the regularity of $D$ is equivalent to the regularity of $w=\left(1-d_{3} d_{3}^{+}\right) d_{2}\left(1-d_{1}^{+} d_{1}\right)$ for one and hence all choices of reflexive inverses $d_{1}^{+}$and $d_{3}^{+}$of $d_{1}$ and $d_{3}$, respectively. Using [14], there is $D^{-}$such that

$$
D D^{-}=\left[\begin{array}{cc}
d_{1} d_{1}^{+} & 0 \\
\left(1-w w^{-}\right)\left(1-d_{3} d_{3}^{+}\right) d_{2} d_{1}^{+} & d_{3} d_{3}^{+}+w w^{-}\left(1-d_{3} d_{3}^{+}\right)
\end{array}\right] .
$$

Consider now the matrix

$$
\begin{aligned}
U & =D A+I-D D^{-} \\
& =\left[\begin{array}{cc}
d_{1} a+1-d_{1} d_{1}^{+} & 0 \\
d_{2} a+d_{3} b-\left(1-w w^{-}\right)\left(1-d_{3} d_{3}^{+}\right) d_{2} d_{1}^{+} & d_{3} d+1-d_{3} d_{3}^{+}-w w^{-}\left(1-d_{3} d_{3}^{+}\right)
\end{array}\right]
\end{aligned}
$$

The existence of $A^{\| D}$ is equivalent to the invertibility of $U$.

Futhermore, using Definition 1.1 together with Theorem 1.3, if $A^{\| D}$ exists then

$$
D\left(V^{-1} A\right) D=A^{\| D} A D=D=D A A^{\| D}=D\left(A U^{-1}\right) D
$$

and therefore $A U^{-1}, V^{-1} A \in D\{1\}$.

Lemma 2.1. Given $M=\left[\begin{array}{cc}m_{1} & 0 \\ m_{2} & m_{3}\end{array}\right]$ with regular diagonal elements, then $M$ has a lower triangular von Neumann inverse if and only if $\left(1-m_{3} m_{3}^{+}\right) m_{2}\left(1-m_{1}^{+} m_{1}\right)=0$.

Proof. Writing $M=\left[\begin{array}{cc}m_{1} & 0 \\ 0 & m_{3}\end{array}\right]+\left[\begin{array}{cc}0 & 0 \\ m_{2} & 0\end{array}\right]=A+B$, let $Y=\left(I-A A^{+}\right) B U^{-1}\left(I-A^{+} A\right)$, with $U=I+A^{+} B=\left[\begin{array}{cc}1 & 0 \\ m_{3}^{+} m_{2} & 1\end{array}\right]$. By [8, Corollary 2.7], $M$ has a lower triangular von Neumann if and only if $Y=0$, that is, $\left(1-m_{3} m_{3}^{+}\right) m_{2}\left(1-m_{1}^{+} m_{1}\right)=0$.

Theorem 2.2. Suppose $R$ is Dedekind-finite and let $A$ and $D$ be as above. Then $A^{\| D}$ exists if and only of $a^{\| d_{1}}$ and $d^{\| d_{3}}$ exist and $\left(1-d_{3} d_{3}^{+}\right) d_{2}\left(1-d_{1}^{+} d_{1}\right)=0$.

In this case,

$$
A^{\| D}=\left[\begin{array}{cc}
a^{\| d_{1}} & 0 \\
v^{-1} d_{2}\left(1-d_{1}^{+}\right) a^{\| d_{1}}+d^{\| d_{3}}\left(b+d_{3}^{+} d_{2} d_{1}^{+}\right) a^{\| d_{1}}+v^{-1} d_{2} & d^{\| d_{3}}
\end{array}\right],
$$

with $v=d_{3} d+1-d_{3} d_{3}^{-}$. 
Proof. Note that if $A^{\| D}$ exists then $U$ is invertible and $A U^{-1} \in D\{1\}$. Since $U$ is a lower triangular invertible matrix and $R$ is Dedekind-finite, then $U^{-1}$ is again lower triangular, and so is $A U^{-1}$. Applying Lemma 1.2 , and since $A U^{-1} \in D\{1\}$, then $w=\left(1-d_{3} d_{3}^{+}\right) d_{2}\left(1-d_{1}^{+} d_{1}\right)=$ 0 . The matrix $U$ then has the form

$$
U=\left[\begin{array}{cc}
d_{1} a+1-d_{1} d_{1}^{+} & 0 \\
d_{2} a+d_{3} b-\left(1-d_{3} d_{3}^{+}\right) d_{2} d_{1}^{+} & d_{3} d+1-d_{3} d_{3}^{-}
\end{array}\right] .
$$

Using the Dedekind-finiteness of $R$, the invertibility of $U$ is equivalent to its diagonal elements being ring units. This in turns means $a^{\| d_{1}}$ and $d^{\| d_{3}}$ exist.

In order to give the expression for $A^{\| D}$, we will compute $U^{-1}$. Setting $u=d_{1} a+1-$ $d_{1} d_{1}^{-}, x=d_{2} a+d_{3} b-\left(1-d_{3} d_{3}^{+}\right) d_{2} d_{1}^{+}$and $v=d_{3} d+1-d_{3} d_{3}^{-}$, the inverse of $U$ is $U^{-1}=$ $\left[\begin{array}{cc}u^{-1} & 0 \\ -v^{-1} x u^{-1} & v^{-1}\end{array}\right]$. This gives

$$
A^{\| D}=U^{-1} D=\left[\begin{array}{cc}
u^{-1} d_{1} & 0 \\
-v^{-1} x u^{-1} d_{1}+v^{-1} d_{2} & v^{-1} d_{3}
\end{array}\right] .
$$

Now

$$
\begin{aligned}
& u^{-1} d_{1}=a^{\| d_{1}}, \\
& v^{-1} d_{3}=d^{\| d_{3}}
\end{aligned}
$$

and

$$
\begin{aligned}
v^{-1} x u^{-1} d_{1}+v^{-1} d_{2} & =v^{-1} d_{2} a^{\| d_{1}}+d^{\| d_{3}} b a^{\| d_{1}}-v^{-1}\left(1-d_{3} d_{3}^{+}\right) d_{2} d_{1}^{+} a^{\| d_{1}}+v^{-1} d_{2} \\
& =v^{-1} d_{2} a^{\| d_{1}}+d^{\| d_{3}} b a^{\| d_{1}}-v^{-1} d_{2} d_{1}^{+} a^{\| d_{1}}+d^{\| d_{3}} d_{3}^{+} d_{2} d_{1}^{+} a^{\| d_{1}}+v^{-1} d_{2} \\
& =v^{-1} d_{2}\left(1-d_{1}^{+}\right) a^{\| d_{1}}+d^{\| d_{3}}\left(b+d_{3}^{+} d_{2} d_{1}^{+}\right) a^{\| d_{1}}+v^{-1} d_{2}
\end{aligned}
$$

which gives the desired expression.

Theorem 2.3. Given a Dedekind-finite regular ring $R$, let $A=\left[a_{i, j}\right]$ and $D=\left[d_{i, j}\right]$ be lower triangular matrices over $R$. If $A^{\| D}$ exists then all $a_{i, i}^{\| d_{i, i}}$ exist and $A^{\| D}$ is again lower triangular.

Proof. Set $d_{i}=d_{i, i}, a_{i}=a_{i, i}$ and write $D=\left[\begin{array}{cc}d_{1} & 0 \\ * & D_{1}\end{array}\right], A=\left[\begin{array}{cc}a_{1} & 0 \\ * & A_{1}\end{array}\right]$. From Theorem 2.2, $a_{1}^{\| d_{1}}$ and $A_{1}^{\| D_{1}}$ exist. Applying the same reasoning to $A_{1}$ and $D_{1}$, we obtain the existence $a_{2}^{\| d_{2}}$. Repeating this process on a finite number of steps, the desired result follows. By induction, and since $A^{\| D}=\left[\begin{array}{cc}a^{\| d_{1}} & 0 \\ * & A_{1}^{\| D_{1}}\end{array}\right]$, then $A^{\| D}$ is again lower triangular.

Applying the previous result to $A^{\| A}=A^{\#}$ and $A^{\| A^{m}}=A^{D}$, we obtain known implications ([15, Proposition 4.2, Corollary 4.1]). That is, given a group invertible [resp. Drazin invertible] 
$n \times n$ matrix $M$ over a Dedekind-finite regular ring, then its diagonal elements are necessarily group invertible [resp. Drazin invertible] and $M^{\#}\left[\right.$ resp. $\left.M^{D}\right]$ is again lower triangular.

Let $R$ be a ring with elements $u, w$ such that $u w=1 \neq w u$. Consider the $2 \times 2$ matrices over $R$ defined as $A=I$ and $D=\left[\begin{array}{cc}u & 0 \\ 1 & w\end{array}\right]$. Note that $D$ is invertible with $D^{-1}=\left[\begin{array}{cc}w & 1-w u \\ -1 & u\end{array}\right]$. Therefore, $A^{\| D}=I$, which is lower triangular, and still $1^{\| u}$ and $1^{\| w}$ do not exist. Indeed, $1^{\| u}$ exists if and only if $u^{\#}$ exists, and $1^{\| w}$ exists if and only if $w^{\#}$ exists. If $u^{\#}$ exists, for instance, then $u^{\#} u^{2}=u$ implies $u^{\#} u=1=u u^{\#}$, that is, $u$ is a unit. A similar reasoning can be applied to $w^{\#}$.

\section{The inverse of a matrix along a lower triangular matrix}

We now consider the inverse of $A=\left[\begin{array}{ll}a & c \\ b & d\end{array}\right]$ along the regular $D=\left[\begin{array}{cc}d_{1} & 0 \\ d_{2} & d_{3}\end{array}\right]$, with $d_{1}, d_{3}$ regular, under a component condition.

Theorem 3.1. For $A$ and $D$ as above such that $a^{\| d_{1}}$ exists then $A^{\| D}$ exists if and only if

$$
\zeta=\beta-\alpha a^{\| d_{1}} c
$$

is a ring unit, where

$$
\begin{aligned}
w & =\left(1-d_{3} d_{3}^{+}\right) d_{2}\left(1-d_{1}^{+} d_{1}\right) \\
\alpha & =d_{2} a+d_{3} b-\left(1-w w^{-}\right)\left(1-d_{3} d_{3}^{+}\right) d_{2} d_{1}^{+} \\
\beta & =d_{2} c+d_{3} d+1-d_{3} d_{3}^{+}-w w^{-}\left(1-d_{3} d_{3}^{+}\right)
\end{aligned}
$$

In this case,

$$
A^{\| D}=\left[\begin{array}{cc}
a^{\| d_{1}} & -a^{\| d_{1}} c \zeta^{-1} d_{3} \\
-\zeta^{-1} \alpha a^{\| d_{1}}+\zeta^{-1} d_{2} & \zeta^{-1} d_{3}
\end{array}\right]
$$

Proof. We know $A^{\| D}$ exists if and only if $U=D A+I-D D^{-}$is invertible. Taking $D^{-}$such that $D D^{-}=\left[\begin{array}{cc}u & 0 \\ \left(1-w w^{-}\right)\left(1-d_{3} d_{3}^{+}\right) d_{2} d_{1}^{+} & d_{3} d_{3}^{+}+w w^{-}\left(1-d_{3} d_{3}^{+}\right)\end{array}\right]$, we obtain

$$
U=\left[\begin{array}{cc}
u & d_{1} c \\
\alpha & \beta
\end{array}\right]
$$

where $u=d_{1} a+1-d_{1} d_{1}^{+}$. Since $a^{\| d_{1}}$ exists, then $u$ is a ring unit, and using Schur complements we may use the factorization

$$
U=\left[\begin{array}{cc}
1 & 0 \\
\alpha u^{-1} & 1
\end{array}\right]\left[\begin{array}{cc}
u & 0 \\
0 & \zeta
\end{array}\right]\left[\begin{array}{cc}
1 & u^{-1} d_{1} c \\
0 & 1
\end{array}\right]
$$


Hence, $U$ is invertible if and only if $\zeta$ is a ring unit.

In order the compute $A^{\| D}$, note that

$$
U^{-1}=\left[\begin{array}{cc}
1 & -u^{-1} d_{1} c \\
0 & 1
\end{array}\right]\left[\begin{array}{cc}
u^{-1} & 0 \\
0 & \zeta^{-1}
\end{array}\right]\left[\begin{array}{cc}
1 & 0 \\
-\alpha u^{-1} & 1
\end{array}\right] .
$$

Then

$$
\begin{aligned}
A^{\| D} & =U^{-1} D \\
& =\left[\begin{array}{cc}
a^{\| d_{1}} & -a^{\| d_{1}} c \zeta^{-1} d_{3} \\
-\zeta^{-1} \alpha a^{\| d_{1}}+\zeta^{-1} d_{2} & \zeta^{-1} d_{3}
\end{array}\right]
\end{aligned}
$$

since $u^{-1} d_{1}=a^{\| d_{1}}$.

Take, as an example, the ring $R$ of $2 \times 2$ matrices over $\mathbb{Z} / 8 \mathbb{Z}$, and its elements

$$
D_{1}=\left[\begin{array}{ll}
7 & 5 \\
1 & 0
\end{array}\right], D_{2}=\left[\begin{array}{ll}
1 & 1 \\
3 & 3
\end{array}\right], D_{3}=\left[\begin{array}{ll}
3 & 6 \\
3 & 6
\end{array}\right]
$$

and

$$
A_{1}=\left[\begin{array}{ll}
5 & 4 \\
1 & 3
\end{array}\right], A_{2}=\left[\begin{array}{ll}
1 & 1 \\
2 & 0
\end{array}\right], A_{3}=\left[\begin{array}{ll}
3 & 6 \\
3 & 6
\end{array}\right], A_{4}=\left[\begin{array}{ll}
1 & 1 \\
6 & 6
\end{array}\right] .
$$

Note that the matrices $A_{1}, D_{1}$ are ring units and $D_{3}$ is idempotent, and as such we can take $D_{3}^{+}=D_{3}$. Consider the matrices over $R$ defined as $D=\left[\begin{array}{cc}D_{1} & 0 \\ D_{2} & D_{3}\end{array}\right]$ and $A=\left[\begin{array}{cc}A_{1} & A_{2} \\ A_{3} & A_{4}\end{array}\right]$. We now follow the notation for $\alpha, \beta, \zeta$ and $w$ of Theorem 3.1. Since $A_{1}, D_{1}$ are ring units then $A_{1}^{\| D_{1}}$ exists and $w=0$. Also,

$$
\alpha=\left[\begin{array}{ll}
5 & 5 \\
7 & 7
\end{array}\right], \beta=\left[\begin{array}{ll}
0 & 2 \\
5 & 5
\end{array}\right], \zeta=\left[\begin{array}{ll}
4 & 4 \\
1 & 3
\end{array}\right] .
$$

Now, the determinant of the matrix $\zeta$ is not relatively prime with 8 , and therefore $\zeta$ is not invertible, which is equivalent to $A^{\| D}$ does not exist.

Using the same notation as in Theorem 3.1, if $d_{1} c=0$, which includes the case $A$ is lower triangular considered in Theorem 2.2, then the matrix $U$ is lower triangular. If the ring is Dedekind-finite then the existence of $A^{\| D}$ implies $a^{\| d_{1}}$ exists.

Corollary 3.2. Let $A=\left[\begin{array}{ll}a & 0 \\ b & d\end{array}\right], D=\left[\begin{array}{cc}d_{1} & 0 \\ d_{2} & d_{3}\end{array}\right]$ with $D, d_{1}, d_{3}$ regular, and suppose $a^{\| d_{1}}$ exists. Then $A^{\| D}$ exists if and only if $d^{\| d_{3}}$ exists and $\left(1-d_{3} d_{3}^{+}\right) d_{2}\left(1-d_{1}^{+} d_{1}\right)=0$.

In this case, $A^{\| D}$ is lower triangular with

$$
A^{\| D}=\left[\begin{array}{cc}
a^{\| d_{1}} & 0 \\
v^{-1} d_{2}\left(1-d_{1}^{+}\right) a^{\| d_{1}}+d^{\| d_{3}}\left(b+d_{3}^{+} d_{2} d_{1}^{+}\right) a^{\| d_{1}}+v^{-1} d_{2} & d^{\| d_{3}}
\end{array}\right],
$$

with $v=d_{3} d+1-d_{3} d_{3}^{-}$. 
Proof. Following the same notation for $U, u, w, \zeta, \beta$ as the proof of the Theorem 3.1, if $U$ is invertible, since it is lower triangular with a unit diagonal element then its inverse is again lower triangular. So, $A U^{-1}$ is a lower triangular von Neumann inverse of $D$, which implies, using Lemma 2.1, that $w=0$. Using Theorem 3.1, $\zeta=\beta=d_{3} d+1-d_{3} d_{3}^{+}$is a unit, that is, $d^{\| d_{3}}$ exists.

For the converse, the matrix $U$ in the proof of Theorem 3.1 has the form

$$
\left[\begin{array}{cc}
d_{1} a+1-d_{1} d_{1}^{+} & 0 \\
* & d_{3} d+1-d_{3} d_{3}^{+}
\end{array}\right]
$$

which is invertible since its diagonal elements are units. Therefore $A^{\| D}$ exists.

From [14], there is

$$
D^{-}=\left[\begin{array}{cc}
1 & 0 \\
-d_{3}^{+} d_{2} & 1
\end{array}\right]\left[\begin{array}{cc}
d_{1}^{+} & \left(1-d_{1}^{+} d_{1}\right) w^{-}\left(1-d_{3} d_{3}^{+}\right) \\
0 & d_{3}^{+}
\end{array}\right]\left[\begin{array}{cc}
1 & 0 \\
-\left(1-d_{3} d_{3}^{+}\right) d_{2} d_{1}^{+} & 1
\end{array}\right],
$$

with $w=\left(1-d_{3} d_{3}^{+}\right) d_{2}\left(1-d_{1}^{+} d_{1}\right)$ for which choice

$$
D^{-} D=\left[\begin{array}{cc}
d_{1}^{+} d_{1}+\left(1-d_{1}^{+} d_{1}\right) w^{-}\left(1-d_{3} d_{3}^{+}\right) d_{2}\left(1-d_{1}^{+} d_{1}\right) & 0 \\
d_{3}^{+} d_{2}\left(1-d_{1}^{+} d_{1}\right)-d_{3}^{+} d_{2}\left(1-d_{1}^{+} d_{1}\right) w^{-}\left(1-d_{3} d_{3}^{+}\right) d_{2}\left(1-d_{1}^{+} d_{1}\right) & d_{3}^{+} d_{3}
\end{array}\right] .
$$

Recall $d^{\| d_{3}}$ exists if and only if $v=d d_{3}+1-d_{3}^{+} d_{3}$ is a ring unit, and $A^{\| D}$ exists if and only if $V=A D+I-D^{-} D$ is an invertible matrix. For the choice of $D^{-}$above,

$$
V=\left[\begin{array}{cc}
\gamma & c d_{3} \\
\eta & v
\end{array}\right]
$$

where

$$
\begin{aligned}
& \gamma=a d_{1}+1-d_{1}^{+} d_{1}-\left(1-d_{1}^{+} d_{1}\right) w^{-}\left(1-d_{3} d_{3}^{+}\right) d_{2}\left(1-d_{1}^{+} d_{1}\right) \\
& \eta=b d_{1}+d d_{2}-d_{3}^{+} d_{2}\left(1-d_{1}^{+} d_{1}\right)+d_{3}^{+} d_{2}\left(1-d_{1}^{+} d_{1}\right) w^{-}\left(1-d_{3} d_{3}^{+}\right) d_{2}\left(1-d_{1}^{+} d_{1}\right)
\end{aligned}
$$

Using Schur complements, we may factor $V$ as

$$
V=\left[\begin{array}{cc}
1 & c d_{3} v^{-1} \\
0 & 1
\end{array}\right]\left[\begin{array}{cc}
\xi & 0 \\
0 & v
\end{array}\right]\left[\begin{array}{cc}
1 & 0 \\
v^{-1} \eta & 1
\end{array}\right]
$$

where $\xi=\gamma-c d_{3} v^{-1} \eta$. Therefore, $V$ is an invertible matrix if and only $\xi$ is a ring unit.

We now compute

$$
\begin{aligned}
V^{-1} & =\left[\begin{array}{cc}
1 & 0 \\
-v^{-1} \eta & 1
\end{array}\right]\left[\begin{array}{cc}
\xi^{-1} & 0 \\
0 & v^{-1}
\end{array}\right]\left[\begin{array}{cc}
1 & -c d_{3} v^{-1} \\
0 & 1
\end{array}\right] \\
& =\left[\begin{array}{cc}
\xi & -\xi c d_{3} v^{-1} \\
-v^{-1} \eta \xi^{-1} & v^{-1} \eta \xi^{-1} c d_{3} v^{-1}+v^{-1}
\end{array}\right]
\end{aligned}
$$


which gives

$$
A^{\| D}=D V^{-1}=\left[\begin{array}{cc}
d_{1} \xi^{-1} & -d_{1} \xi^{-1} c d^{\| d_{3}} \\
d_{2} \xi^{-1}-d^{\| d_{3}} \eta \xi^{-1} & -d_{2} \xi^{-1} c d^{\| d_{3}}+d^{\| d_{3}} \eta \xi^{-1} c d^{\| d_{3}}+d^{\| d_{3}}
\end{array}\right]
$$

since $d^{\| d_{3}}=d_{3} v^{-1}$.

We have proved the following result:

Theorem 3.3. For $A, D, \eta, v, w$ as above such that $d^{\| d_{3}}$ exists then $A^{\| D}$ exists if and only if

$$
\xi=\gamma-c d^{\| d_{3}} \eta
$$

is a ring unit. In this case,

$$
A^{\| D}=D V^{-1}=\left[\begin{array}{cc}
d_{1} \xi^{-1} & -d_{1} \xi^{-1} c d^{\| d_{3}} \\
d_{2} \xi^{-1}-d^{\| d_{3}} \eta \xi^{-1} & -d_{2} \xi^{-1} c d^{\| d_{3}}+d^{\| d_{3}} \eta \xi^{-1} c d^{\| d_{3}}+d^{\| d_{3}}
\end{array}\right] .
$$

Corollary 3.4. Let $A=\left[\begin{array}{ll}a & 0 \\ b & d\end{array}\right], D=\left[\begin{array}{cc}d_{1} & 0 \\ d_{2} & d_{3}\end{array}\right]$ with $D, d_{1}, d_{3}$ regular, and suppose $d^{\| d_{3}}$ exists. Then $A^{\| D}$ exists if and only if a ${ }^{\| d_{1}}$ exists and $\left(1-d_{3} d_{3}^{+}\right) d_{2}\left(1-d_{1}^{+} d_{1}\right)=0$.

Proof. If $a^{\| d_{1}}$ exists and $\left(1-d_{3} d_{3}^{+}\right) d_{2}\left(1-d_{1}^{+} d_{1}\right)=0$, since $d^{\| d_{3}}$ exists then using Corollary 3.2 the existence of $A^{\| D}$ follows.

Suppose now $A^{\| D}$ and $d^{\| d_{3}}$ exist. Then the matrix $V$ defined in (1) is a lower triangular matrix with invertible diagonal elements. Therefore $V^{-1}$ is invertible. Since $V^{-1} V^{-1} A \in D\{1\}$ then $\left(1-d_{3} d_{3}^{-}\right) d_{2}\left(1-d_{1}^{-} d_{1}\right)=0$.

\section{Remarks}

Theorem 1.3 collapses to known characterizations of group and Moore-Penrose invertibility. Indeed, when $a=1$ then we obtain [17], and when $a=d$, we obtain an equivalent condition to [18], since $d^{3} d^{-}+1-d d^{-}$is a unit if and only if $d^{2}+1-d d^{-}$is a unit, by Lemma 1.2 and by $d^{3} d^{-}+1-d d^{-}=\left(d^{2} d^{-}+1-d d^{-}\right)^{2}$. It could be of interest to link to the characterization of group invertibility given in [11]. When $a=d^{*}$ we obtain the characterization given in [16]. Finally, it is not clear how to relate the inverse along an element with the adugate of an operator matrix, as defined in [6].

\section{Acknowledgment}

The authors wish the thank both referees for their corrections and remarks. 


\section{References}

[1] Ben Israel, A.; Greville, T. N. E.; Generalized Inverses, Theory and Applications, 2nd Edition, Springer, New York 2003.

[2] Brown, B.; McCoy, N. H.; The maximal regular ideal of a ring. Proc. Amer. Math. Soc. 1, (1950), 165-171.

[3] Castro-González, N.; Mendes-Araújo, C.; Patrício, P.; Generalized inverses of a sum in rings. Bull. Aust. Math. Soc. 82 (2010), no. 1, 156-164.

[4] Cvetković-Ilić, D.; Harte, R.; On Jacobson's lemma and Drazin invertibility, Appl. Math. Lett., 23 (2010), no. 4, 417-420.

[5] Green, J.A.; On the structure of semigroups, Ann. of Math., 54 (1951), no. 1, 163-172.

[6] Harte, R.E.; Hernandez, C.; Adjugates in Banach algebras. Proc. Amer. Math. Soc. 134 (2005), 1397-1404.

[7] Hartwig, R.E.; Block generalized inverses, Arch. Rational Mech. Anal. 61 (1976), no. 3, $197-251$.

[8] Hartwig, R.E.; Patrício, P.; Puystjens, R.; Diagonalizing triangular matrices via orthogonal Pierce decompositions. Linear Algebra Appl. 401 (2005), 381-391.

[9] Hartwig, R.E.; Shoaf, J.; Group inverses and Drazin inverses of bidiagonal and triangular Toeplitz matrices. J. Austral. Math. Soc. Ser. A, 24 (1977), no. 1, 10-34.

[10] Huylebrouck, D.; Puystjens, R. Generalized inverses of a sum with a radical element. Linear Algebra Appl. 84 (1986), 289-300.

[11] Kantun-Montiel, D.; Djordjević, S.V.; Harte, R.E., On semigroup inverses. Funct. Anal. Approx. Comp. 1 (2009), 11-18.

[12] Mary, X.; On generalized inverses and Green's relations. Linear Algebra Appl. 434 (2011), no. $8,1836-1844$.

[13] Mary, X; Patrício, P.; Generalized inverses modulo $\mathcal{H}$ in semigroups and rings, submitted for publication.

[14] Patrício, P.; Puystjens, R.; About the von Neumann regularity of triangular block matrices. Linear Algebra Appl. 332/334 (2001), 485-502.

[15] Patrício, P.; Hartwig, R. E. Some additive results on Drazin inverses. Appl. Math. Comput. 215 (2009), no. 2, 530-538.

[16] Patrício, Pedro; Mendes-Araújo, C.; Moore-Penrose invertibility in involutory rings: the case $a a^{\dagger}=b b^{\dagger}$. Linear Multilinear Algebra 58 (2010), no. 3-4, 445-452. 
[17] Patrício, Pedro; Veloso da Costa, Antnio; On the Drazin index of regular elements. Cent. Eur. J. Math. 7 (2009), no. 2, 200-205.

[18] Puystjens, R.; Hartwig, R. E.; The group inverse of a companion matrix. Linear and Multilinear Algebra, 43 (1997), no. 1-3, 137-150, 